\title{
Contents, Vol. 6, No. 4, 1996
}

\section{Inhalt $\square$ Contents}

Verhaltenstherapie

Vol. 6, Nr. 4, Dezember 1996

Vol. 6, No. 4, December 1996

Originalarbeiten

Original Papers

201 Stationäre ßehandlung von Patienten mit Doppeldiagnosen

Moggi, F.; Hirsbrunner, H.-R; Wittig. R.; Donati. R.; Brodbeck. I; Bachmann, K.M. (Bern)

210 «Born to be wild oder doch gelernt?» Neue verhaltensmedizinische Erkenntnisse zur Ätiopathogenese der Migräne

Gerber, W. D.; Kropp, P. (Kiel); Schoenen, J. (Liege); Siniatchkin, M.S. (Moskau)

222 Ecstasy- und Halluzinogengebrauch bei Jugendlichen Gibt es eine Zunahme?

Schuster. P.: Wittchen, H.-U. (München)

201 Inpatient Treatment of Patients with Dual Diagnoses

Moggi, F.; Hirsbrunner. H.-R; Wittig. R.; Donati. R.; Brodbeck, J.; Bachmann, K.M. (Bern)

210 'Born to be Wild or Learned?' New Vistas to

the Aetiopathogenesis of Migraine Derived from Behavioural Medicine

Gerber, W.D.; Kropp, P. (Kiel); Schoenen, J. (Liege); Siniatchkin. M.S. (Moskau)

222 Ecstasy and Hallucinogene Use in Adolescence on the Rise?

Schuster. P.; Wittchen, H.-U. (München) 
Falldarstellung

Case Report 
234 «ldeot Savant» ohne Autismus: Die Entwicklung eines geistig behinderten Kindes mit Spezialbegabung von 8 Monaten bis 8 Jahren Süss-Burghart, H. (München)

234 'Ideot Savant' without Autism: The Development of a Mentally Retarded Child with a Special Talent from 8 Months to 8 Years of Life Süss-Burghart, H. (München)

\section{Diskussionsforum}

\section{Forum}

244 Individualisierung statt Standardisierung:

Verhaltenstherapie als biographisch orientierte Neuerfahrung

Zarbock, G. (Hamburg)

244 Individualization Instead of Standardization: Behavior Therapy as Biographically Oriented Corrective Experience Zarbock, G. (Hamburg) 
252 Neuigkeiten vom Buch- und Zeitschriftenmarkt 252 Articles and Books 
258 Mitteilungen der Verbände

258 Institut für Verhaltenstherapie Berlin e.V., Berlin

258 Institut für Therapieforschung (IFT)

258 Vereinigung der Kassenpsychotherapeuten

Arbeitsgemeinschaft für Verhaltungsmodifikation-Schweiz (AVM-CH)

Eifeler-Verhaltenstherapie-Institut e.V. (EVI) 

269 Meetings and Conferences 

198 Impressum

Autorenverzeichnis 1996

Sachwortverzeichnis 1996 Jahres-Inhalt 1996 in (nach 276)

U3 Hinweise für Autoren (3. L1/2schiagseite) 
198 Imprint

274 Author Index 1996

276 Subject Index 1996

Complete Contents 1996 iv (following 276) 

(C) 1996 S. Karger GmbH. Freiburg

Fax (0761)45207 14

http:/www.karger.ch/journals/ver/verdes.htm 
Das Altern von Menschen und $\mathrm{T} ;$ eren, neuartig evolutionsbiologisch begründet 
Altern

Ergebnis

ökologischer

Anpassung 
Alternstheorien dieses Jahrhunderts ¡m kritischen Überblick 
Inhalt

Zum Begriff Seneszenz

Kriîischer Überblïckzu mass-

geblichen Altemstheor;en dieses Jahrhunderts

Leben, Strukturerhaltung und Reproduktion

Seneszenz-ökologisch zweckmässig

Die Selektionsfaktoren der Lebensdauer

BeziehungderPhysiologie des Seneszenzverlaufs zum Reprodukt;onswert

Zurmoiekularbiologischen Regelung des Seneszenzverlaufs im Individuum

Schlussfolgerungen

Zusammenfassung 
Die Suche ach den Ursachen des biologischen Alterns (Seneszenz) hat noch z keiner allgemein akzeptierten Theorie des Alterns geführt. Bisherige Theorien sin überwiege $\cap d$ gekennze; dieses Buche diskutiert zunächst grundsätzliche Schwächen solcher «destruktiv-entropische Alternstheorien», bevor er die Evolution von artspezifischer Lebensdauer un Seneszenz auf adaptiver Grundlage erörtert. Als von eीtscheidender Bedeutun für die Evolution der artspezifischen Lebensdauer werden die Grenzen der ökolog schen Kapazität und der Zeitbedarf für die Optjmierung des Fortpflanzungsprozes ses hervorgehoben. Dabei wird das Altern des Individuums - auch des Menschen als ein biologisch sinnvolies und abgestimmtes Geschehen zur Erhaltung der Ai deutlich.

Mit der steigenden Lebenserwartung ist auch eine Zunahme der alterstypische Krankheiten verbunden. Für den Arzt ist daher das Verständnis und Erkenne von normalen «physiologischen» Funktionseinschränkungen im Altemsprozess un eine Abgrenzung gegenüber «altemsbegle ¡tenden Krankheiten» sehr wichtig. Dieses Buch leistet dazu einen wesentlichen Beitrag, indem es - auch biologisch medizinisch Interessierte - mit den theoretischen Grundlagen und biologische Mechanismen von Altemsveränderungen vertraut macht. 

Ahlert, G. (Qberursel)

Altern - Ergebnis ökologischer Anpassung

Alternstheorien dieses Jahrhunderts $¡ m$ kritischen Überbl $; c k$

$x+68$ S., 2 Abb., 2 Tab., broschiert, 1996

CHF57.-/DEM68.-/USD 49.75

Preisäกderungen vorbehalten

DEM-Preis nur für Deutschiand, USD-Preis nur für USA

ISBN 3-8055-6361-2 
Zahlung:

Bitte sender) Sie:

— Expl.; Ahlert, G.: Altern - Ergebnis ökologischer Anpassung

Alternstheorien dieses Jahrhunderts im kritischen Ûberblick CHF 57.-/DEM 68.-/USD 49.75 ISBN 3-8055-6361-2

a Informationen zu Karger Publikationen in verwandten Fachgebieten 
Bei Vorauszahlung Porto und Verpackung zu Lasten des Verlî

a Scheck be ¡liegend

$\square$ Rechnung

o Diners

$\propto$ Kreditkarte (DEM werden in CHF belastet)

a American Express D Visa

a MasterCardo Eurocard 
Karte-Nr.:

Gültig bis:

IMame/Adresse: 
S. Karger AG

Postfach, CH-4009 Basel Fax+41 613061234 E-Mail karger@karger.ch http://www.karger.ch 
S. Karger $\mathrm{GmbH}$

Postfach

D-79095 Freiburg Fax (0761)45 20174 
